\title{
Implantación sindical y participación electoral en la Segunda República: Cádiz (1933-1936)
}

\section{DIEGO CARO CANCELA}

Quizá una de los aspectos mejor tratados de la historiografía contemporánea sea el de la sociología electoral de la Segunda República. Desde los trabajos pioneros de Jean Bécarud (1) ó Javier Tusell (2), han sido numerosas las monografias dedicadas a las elecciones republicanas en los ámbitos local, provincial o regional, con metodologías más o menos parecidas o a veces repetidas mecánicamente.

Un elemental análisis de esta ya amplia bibliografía permite hacer un somero balance de lo publicado y distinguir dos etapas claramente diferenciadas. Hay un primer momento de estudios generales, en el que los trabajos apenas si superan el tradicional "patron" metodologico de la trilogía "candidaturas-campañas-resultados", sin avanzar más. Sin embargo, ya a finales de los años setenta, las nuevas investigaciones, normalmente bajo el trámite académico de tesina o tesis, ofrecen un horizonte más amplio. No se limitan a ser una exposición meramente descriptiva del panorama electoral y sus resultados, sino que avanzan sobre líneas más renovadoras, en las que las opciones eléctorales aparecen como la última consecuencia de distintas variables, como la distribución de la tierra, el tamaño de los municipios, la estructura socioprofesional o un concreto sistema de partidos (3).

Esta manera, más "global", de hacer sociología electoral, presta tanta atención a los votantes, como a los abstencionistas, sobre todo, teniendo en cuenta que una de las principales fuerzas sociales del

(1) BECARUD, Jean: La Segunda República Fspañola, 1.931-1.936. Ensayo de interpretación. Madrid, 1.967.

(2) TUSELL, Javier: La Segunda República en Madrid: elecciones y partidos políticos. Madrid, 1.970. O también: Las elecciones del Frente Popular. Madrid, 1.971.

(3) Un buen ejemplo en: GERMAN, Luis: Aragón en la Segunda República. Estructura económica y comportámiento político. Zaragoza, 1.984.

DOI: http://dx.doi.org/10.25267/Trocadero.1993.i5.33 
régimen republicano -los anarcosindicalistas de la CNT- juega un papel clave en la España de los años treinta, caracterizada por una movilización política sin precedentes en la vida pública (4).

Es en este análisis del fenómeno electoral más explicativo en el que pretendemos situar el presente trabajo, dedicado al estudio de lo que ya se ha llamado "el abstencionismo intermitente", influenciado ideológicamente por el anarquismo, en contraposición a un abstencionismo estructural de múltiples variantes y motivaciones, como es el que se da en esta misma época en territorios como Galicia y Canarias.

Hace algunos años, Javier Tusell planteaba como un reto para la nueva sociología electoral republicana, el afrontar la medición de las variaciones de esta intermitencia, ya estudiada en algunas zonas de Cataluña por Mercedes Vilanova (5), quedando pendiente la comparación con el otro gran bastión del anarquismo peninsular, el foco andaluz. Pues bien, con esta pretensión ofrecemos los siguientes datos y comentarios, centrados en el marco geográfico de la provincia de Cádiz, dejando para otro momento y lugar el análisis del abstencionismo republicano en el conjunto de la Andalucía anarcosindicalista.

En la elección de Cádiz contamos con dos ventajas previas sobre otros distritos andaluces. En primer lugar, a diferencia de las provincias de Sevilla o Málaga -por citar dos como ejemplo-, aquí el conjunto de la provincia forma una única circunscripción electoral, incluida su propia capital. Por otra parte, el territorio gaditano es, de toda la región, la zona en la que de forma más clara se manifiesta la hegemonía de la CNT en el conjunto del movimiento obrero y más perceptible, por tanto, 'su influencia (6).

Para demostrar nuestra hipótesis de trabajo - la incidencia determinante de la actitud de la CNT en los resultados-, haremos una comparación de las elecciones de 1933 y 1936, siguiendo respecto al número de votos recibidos por las candidaturas y el cálculo del abstencionismo, la metodología empleada anteriormente en nuestro libro $L a$ Segunda República en Cádiz: elecciones y partidos políticos (7), al que

(4) Sobre la actuación y la estrategia de la CNT en la II República, vid: BRADEMAS, John: Anarcosindicalismo y revolución en España, 1.930-1.937. Barcelona, 1.974.

(5) VILANOVA, Mercedes: "El abstencionismo electoral y su relación con las fuerzas políticas en la provincia de Gerona durante la II República" en Homenaje al profesor Regla. Valencia, 1.975.

(6) Un sencillo manual sobre la legislación electoral republicana en: BARREIRO DE W. Y NOYA, Augusto(coordinador): Ley Electoral Vigente para diputados a Cortes $y$ Concejales. La Coruña, 1933.

(7) Cádiz, 1.987. 
también remitimos para conocer más detalladamente el contexto político provincial en el que se realizan ambas consultas electorales, el proceso de formación de listas seguidos en cada caso y, finalmente, los resultados completos por municipios.

\section{LA REALIDAD DE LAS ORGANIZACIONES OBRERAS GADITANAS}

Un primer problema nos encontramos a la hora de intentar aproximarnos al conocimiento de la implantación geográfica de las dos grandes fuerzas sindicales de la época -la CNT y la UGT-, en el marco de la provincia de Cádiz: la falta de estadísticas coetáneas para ambos sindicatos, por las dispares fechas en las que celebran sus Congresos nacionales; inconveniente que se acentúa en el caso de la UGT por la ausencia de referencias a la distribución de militantes por municipios en sus documentos congresuales de 1932, por lo menos, en los que hemos podido consultar. De aquí que tengamos que suplir este obstáculo con los datos que ofrece la Memoria del II Congreso de la FNTT, que acumula interesantes informaciones sobre la distribución territorial de este sindicalismo campesino ugetista, que, por otra parte, era el único que realmente existía en muchísimos municipios andaluces y españoles del mundo rural, mientras que para conocer la implantación cenetista acudiremos a las estadísticas de las Regionales que participaron en el Congreso confederal de Zaragoza de mayo de 1936, más completas para nuestro trabajo que las del Congreso de 1931, cuando la CNT se encontraba en pleno proceso de reconstrucción, después de la forzada clandestinidad de la Dictadura y apenas había empezado a experimentar el importante crecimiento del período republicano.

Una primera conclusión salta a la vista, después de comparar estos datos: la CNT era, con enorme diferencia, la principal fuerza sindical de Cádiz, en donde tenía uno de sus principales bastiones de Andalucía, después de Sevilla, multiplicando casi por cuatro su número de afiliados entre 1931 y 1936, pasando ya en 1932, de ser la cuarta a la segunda provincia andaluza. El contraste con la implantación ugetista no puede ser más evidente en el conjunto del contexto andaluz:

NUMERO DE AFIIIADOS

CNT (1932) . UGT (1931)

$\begin{array}{lcr}\text { Almería } & 5.000 & 12.923 \\ \text { Cádiz } & 34.842 & 7.722 \\ \text { Córdoba } & 32.038 & 17.585\end{array}$




\section{CNT (1932) UGT (1931)}

$\begin{array}{lrr}\text { Granada } & 25.2 \mathrm{O} 2 & 15.060 \\ \text { Huelva } & 14.955 & 13.581 \\ \text { Jaén } & 9.982 & 20.527 \\ \text { Málaga } & 34.225 & 17.485 \\ \text { Sevilla } & 140.000 & 19.984\end{array}$

Fuente: Boletín UGT de España, núm. 36, 1931 CNT(25-XI-1932), núm. 11.

Al margen de lo discutible que pueden ser algunas de las cifras anteriores, proporcionadas por el periódico CNT, la importancia del anarcosindicalismo gaditano es más que evidente, hasta alcanzar en mayo de 1936 los 45.323 afiliados, con una implantación repartida por casi toda la geografía provincial, incluida la comarca serrana de tradicional hegemonía ugetista.

El predominio de las sociedades adscritas a la CNT era manifiesto en los principales núcleos urbanos de la provincia, como Cádiz, Jerez, San Fernando, Algeciras, La Línea o Sanlúcar. Y si en estas poblaciones, la presencia anarquista tenía unas hondas raíces históricas, ahora, durante el régimen republicano, la central anarcosindicalista también se extendía a municipios donde nunca había tenido representación como Chiclana, Vejer, Tarifa, Villamartín o Puerto Serrano, todos ellos de tamaño medio-pequeño, mientras mantenía sus tradicionales posiciones serranas en Ubrique y Grazalema (8).

Este incremento de la militancia en el sindicato cenetista no sólo se debió a la importante movilización en la vida sindical y política que provocó el cambio de régimen, con un incremento generalizado de militantes y organizaciones obreras, sino también al ingreso en las filas anarcosindicalistas de sociedades que hasta entonces habían estado adheridas a la UGT. Son los casos, por poner algunos ejemplos, del Centro Instructivo de Obreros del Campo, de Trebujena, de las sociedades de panaderos, arrumbadores, ferroviarios y viticultores de Jerez o de la socialista Casa del Pueblo de El Gastor. Un trasvase que se explicaría por los fracasos de la política reformista del primer Gobierno republicano-socialista y el consiguiente desencanto que crea en los medios obreros y también por la propia práctica sindical de la CNT, que ahora parecía como más adecuada a los tiempos de crisis y de agudización de los conflictos de

(8) Más datos sobre la realidad sindical de la provincia de Cádiz en: VV.AA.: El movimiento obrero en la bistoria de Cádiz. Cádiz, 1.988.

612 
clase. De todas formas, estas son explicaciones que necesitarían completarse con otras motivaciones más de tipo local, porque lo cierto es que también en estos años de 1931 a 1933, la UGT experimenta una notable expansión en el medio rural, merced a la organización de los trabajdores del campo en la FNTT, que en Cádiz pasa de 8 secciones en febrero de 1932, a 15 cuatro meses más tarde, casi todas ellas localizadas en la serranía gaditana, al noreste de una provincia que seguía siendo la última de Andalucía, pese al crecimiento experimentado (9):

\section{EFECTIVOS DE LA FNTT DE LA UGT}

\section{SECCIONES}

$\begin{array}{lr}\text { Almería } & 84 \\ \text { Cádiz } & 15 \\ \text { Córdoba } & 64 \\ \text { Granada } & 32 \\ \text { Huelva } & 52 \\ \text { Jaén } & 93 \\ \text { Málaga } & 87 \\ \text { Sevilla } & 40 \\ \text { TOTAL ............ } & 467\end{array}$

\section{FEDERADOS}

10.920

21.003

10.184

9.191

32.633

21.120

15.397

125.617

Esta débil implantación del sindicalismo ugetista en el conjunto del movimiento obrero gaditano, se acentuaba en el caso del PSOE, que no sólo no incrementaba sus afiliados de 1931 a 1932, sino que pierde más de mil -al pasar de 2.162 a 977-, en una anómala situación, que Gérard Brey justifica en el hecho de que los socialistas en Cádiz privilegiaron más la reorganización sindical que el proselitismo político, en un sentido estricto. De esta manera, se daba el caso que había poblaciones serranas de clara hegemonía ugetista que no tenían agrupación del PSOE, como El Bosque, Alcalá del Valle o Algar o que hubiera que esperar hasta el 8 de abril de 1933 para que se pudiera celebrar el Primer Congreso de Agrupaciones Socialistas de la provincia de Cádiz, con delegados de poco más de una cuarta parte de los municipios. Y todavía más escasa era la influencia del movimiento comunista, en contraste con su pujanza en otras provincias andaluzas, como Córdoba o Sevilla. Sólo tenía un concejal en el Ayuntamiento serrano de Algodonales y ni siquiera había podido presentar candidatura en las Constituyentes de 1931.

(9) Un completo análisis de la evolución de la FNTT en la II Repúbliça en:TUÑON DE LARA, Manuel: Tres claves de la II Repuiblica. Madrid, 1.985, pp. 21-218. 
Su principal núcleo estaba en El Puerto de Santa María, donde ejercía una importante actividad sindical, gracias al trabajo de líderes como Juan Modesto o Daniel Ortega. De aquí y en el transcurso de la Segunda República, su implantación se extendió a poblaciones vecinas, hasta alcanzar entre 1933 y 1934, la ridícula cifra de 168 afiliados, repartidos en 22 células de nueve poblaciones de la provincia (10).

Hecha esta elemental aproximación al movimiento obrero gaditano y sus organizaciones, un análisis de las estadísticas disponibles de la CNT y de la FNTT de la UGT, sobre el número y la localización de las sociedades que tenían adheridas, completado con otras informaciones indirectas que hemos conseguido, nos permite clasificar a los 41 municipios de Cádiz, en los tres grupos siguientes:

\section{HEGEMONIA \\ DE LA CNT}

Algeciras

Alcalá de los Gazules

Arcos de la Frontera

Los Barrios

Castellar de la Frontera

Chiclana de la Frontera

El Gastor

Cádiz

Jimena de la Frontera

La Línea

Jerez de la Frontera

Medina-Sidonia

Paterna de Rivera

Puerto Real

Puerto Serrano

Sanlúcar de Barrameda

San Roque

San Fernando

Tarifa

Trebujena

Ubrique

Vejer de la Frontera

Villaluenga del Rosario

Grazalema

\section{HEGEMONIA}

\section{DE LA UGT}

Alcalá del Valle

Algar

Benaocaz

Bornos

El Bosque

Chipiona

Espera

Olvera

Setenil

El Puerto de Santa María

Villamartín

Zahara de la Sierra

Torre-Alháquime

\section{SIN DATOS}

Algodonales

Conil

Prado del Rey

Rota 


\section{ABSTENCIONISMO Y VICTORIA DE LAS DERECHAS EN $19 \dot{3} 3$}

Rota la coalición republicano-socialista que había traído el nuevo régimen $\mathrm{y}$ desprestigiado el Gobierno por los sucesos de Casas Viejas y el estancamiento de su programa de reformas, el Presidente de la República decide retirarle su confianza y convocar nuevas elecciones legislativas para el 19 de noviembre de 1933, cuando ya habían culminado los trabajos de reorganización de las derechas alrededor de la CEDA, con el Partido Radical cada vez más escorado hacia posiciones conservadoras y un socialismo que empezaba a resquebrajarse abiertamente entre moderados "prietistas" y un ala "caballerista" que tomaba posiciones en el seno de la UGT.

En Cádiz, a estas elecciones de 1933 se presentaban cuatro listas para los ocho puestos asignados a la mayoría, mientras que dos quedaban reservados a la minoría. Una era la llamada Candidatura de la Unión Ciudadana y Agraria, formada por las figuras más representativas del monarquismo provincial, la lista del Partido Radical, que lideraba el abogado gaditano Santiago Rodríguez-Piñero en la de la coalición de izquierda se integraban los más conocidos dirigentes del socialismo provincial y el republicanismo de izquierda y, finalmente, concurrían por primera vez en la provincia de Cádiz a unas elecciones, los comunistas del PCE, bajo la denominación del Frente Unico Revolucionario.

Ante estos comicios, el tradicional abstencionismo del movimiento anarcosindicalista se ve reforzado ahora por la abierta confrontación que la CNT -controlada por los "faístas"- había mantenido con el gobierno republicano-socialista, plasmada en las dos insurrecciones de enero de 1932 y 1933 y saldadas con un balance de numerosos muertos en los enfrentamientos, cientos de detenidos y una represión que alcanzaba su máxima expresión simbólica en lo ocurrido en la aldea de Casas Viejas, en la provincia de Cádiz.

Totalmente desencantados pues, con el gobiemo republicano de izquierda, los cenetistas desarrollan una campaña, pidiendo la abstención, en la que incluso participa desde la cárcel el líder sindical gaditano Vicente Ballester, desde las páginas del diario madrileño confederal $C N T-A I T$, con variados mensajes y consignas, en los que el tema central era la descalificación de todos los políticos y los partidos, como personajes corruptos y malhechores, cualquiera que fuera su ideología:

"Obrero: las derechas te asesinarán; las izquierdas te fusilarán; el fascismo te apunaalará. Todos te encadenarán y te explotarán" (11).

(11) CNT-AIT(2-XI-1.933). 
O como este otro, comparando los programas:

"Programa de la derecha: Explotación del hombre por el hombre. Programa de las izquierdas: Explotación del hombre por el hombre. ¡ Pueblo: aplasta a la política!(...) (12).

Después de una campaña plagada de incidentes, con atentado terrorista incluido, el 19 de noviembre se desarrollaban las votaciones con absoluta normalidad. Al día siguiente, ya las primeras noticias que recogía la prensa sobre las jornada electoral, eran la prueba más palpable de que se había producido un fuerte abstencionismo. Así, el Diario de Cádiz, el 20 de noviembre, en su edición de mañana, contrastaba la considerable mayoría de mujeres en las colas formadas frente a los colegios, con sacerdotes y algunas religiosas incluidas, con "las grandes masas de electores absolutamente indiferentes a la función ciudadana que se desarrollaba", por el Campo del Sur y los extramuros de la capital. Más claramente, el diario gaditano escribía que, "los elementos sindicalistas afiliados a la CNT y la FAI obedecieron de modo absoluto la consigna ordenada por sus dirigentes y se abstuvieron totalmente de votar".

Lo mismo decía el corresponsal del periódico en Sanlúcar, el día 2l, al anotar que, "la abstención de la CNT fue absoluta". Y en Jerez, semejante impresión sacó José Antonio Primo de Rivera de lo que ocurría, cuando bien temprano visitó uno de los colegios de la ciudad y comprobó, "como los votantes de derechas acudían todos a las urnas y que la CNT cumplía la abstención anunciada" (13).

Al final, hecho el recuento en el conjunto de la provincia, de un censo de 264.694 electores, sólo llegaron a votar 94.654 , lo que representaba el 37,27 por ciento y daba un porcentaje de abstención del 62,73 por ciento, que era el más alto de toda España, seguido por los otros dos distritos andaluces de Málaga y Sevilla provincia.

Pero este elevado abstencionismo no se repartía de forma uniforme por toda la geografía provincial, sino que como pretendemos demostrar a continuación, tenía una relación muy directa con la implantación sindical existente en los diferentes municipios gaditanos, señal inequívoca de que los cenetistas habían seguido masivamente la consigna "¡Obreros, no votéis!", de sus dirigentes.

(12) CNT-AIT(16-XI-1.933).

(13) DAVILA, Sancho-PEMARTIN, Julián: Hacia la bistoria de la falange. Primera contribución de Sevilla. Jerez, 1.938, p. 51. 
Con esta finalidad, vamos a ordenar de mayor a menor estas poblaciones, según sus índices de abstención, señalando al lado la central hegemónica. Como puede comprobarse en el CUADRO I, en los 15 municipios con un abstencionismo superior al 70 por ciento, el predominio sindical corresponde a la CNT, exceptuando Bornos, donde la sociedad obrera local pertenecía a la ugetista FNTT y encabezaba la relación precisamente Paterna de Rivera, localidad en la que vivía Miguel Pérez Cordón, uno de los principales líderes del sindicalismo campesino anarquista y colaborador habitual en la prensa de esta ideología.

\section{CUADRO I}

EL ABSTENCIONISMO EN 1933 (MAS DEL 70 \%)

\section{MUNICIPIO ABSTENCION HEGEMONIA LISTA MAS SINDICAL VOTADA}

\begin{tabular}{|c|c|c|}
\hline Paterna & 85,2 & $\mathrm{CNT}$ \\
\hline El Gastor & 82,0 & CNT \\
\hline Castellar & 80,4 & $\mathrm{CNT}$ \\
\hline Los Barrios & 79,8 & $\mathrm{CNT}$ \\
\hline Alcalá Gazules & 79,8 & $\mathrm{CNT}$ \\
\hline Algeciras & 79,6 & $\mathrm{CNT}$ \\
\hline San Roque & 78,3 & $\mathrm{CNT}$ \\
\hline Sanlúcar & 76,9 & $\mathrm{CNT}$ \\
\hline Bornos & 76,7 & UGT \\
\hline Medina-Sidonia & 75,5 & CNT \\
\hline La Línea & 75,4 & $\mathrm{CNT}$ \\
\hline Arcos & 74,7 & CNT \\
\hline Grazalema & 73,6 & $\mathrm{CNT}$ \\
\hline Jimena & 73,4 & $\mathrm{CNT}$ \\
\hline Ubrique & 71,8 & CNT \\
\hline
\end{tabular}

Por contra, en las 15 poblaciones con índices de participación superiores al 40 por ciento, como se puede comprobar en el CUADRO II, los de manifiesta hegemonía ugetista son mayoría, liderando la relación municipios en donde esta presencia también se ve acompañada por la existencia 
de una sólida Agrupación Socialista, como son los casos de El Puerto, Setenil, Villamartín, Espera, Olvera o el propio San Fernando, aunque en este último caso, la participación se pueda explicar también por la clara tendencia derechista de una parte significativa de su electorado, vinculado a la presencia de contigentes militares en los acuartelamientos de la ciudad.

\section{CUADRO II \\ LA PARTICIPACION EN 1933 (MAS DEL $40 \%$ )}

\section{MUNICIPIO PARTICIPACION}

El Bosque

Torre-Alháquime

El Puerto Sta. Ma.

Setenil

Villamartín

Algodonales

Rota

Tarifa

Chipiona

Prado del Rey

Conil

Espera

Olvera

San Fernando

Benaocaz
84,2
74,1
64,2
63,0
62,0
60,1
56,3
55,6
55,2
52,1
50,8
49,5
47,7
41,9
41,7
HEGEMONIA SINDICAL

UGT

UGT

UGT

UGT

UGT

?

?

CNT

UGT

?

?

UGT

UGT

CNT

UGT LISTA MAS
VOTADA

I

I

I

I

I

$\mathrm{R}$

D

D

D

I

I

$\mathrm{R}$

D

D

D

Por tanto, como primera conclusión que se puede extraer, parece evidente que la abstención se ha producido, sobre todo, en los municipios controlados sindicalmente por la CNT y en mucho menor medida en los que no estaban bajo su órbita de influencia.

La siguiente cuestión a resolver era si esta inhibición del movimiento obrero anarcosindicalista propició o no el triunfo de la candidatura de derechas en estas elecciones. Sobre el particular, las opiniones están divididas. Para Tuñón de Lara, por ejemplo, el abstencionismo anarquista mermó considerablemente los votos de las izquierdas (14), criterio que también comparte Gerald Brenan

(14) TUÑON DE LARA, Manuel: "La Segunda Repüblica" en La crisis del Estado: Dictadura, República, Guerra(1.923-1.939). Tomo IX de la Historia de España, dirigida por ... Barcelona, 1.981, pp. 171-172. 
(15); incluso Richard Robinson llega a cuantificar en 800.000 los votos proletarios que perdió la izquierda en toda España con la decisión abstencionista de la CNT (16).

No es de esta opinión José Macarro, quien en su excelente tesis sobre el movimiento obrero sevillano, al analizar estos comicios, escribe que, "la importancia atribuída a la abstención de la CNT es un mito", porque no tuvo nada que ver con el triunfo de las derechas (17).

De todas formas, veámos lo que se dijo entonces. El periódico $\mathrm{El}$ Noticiero Gaditano, portavoz oficioso de la azañista Acción Republicana lo tenía claro al resumir la información que dedicaba a la jornada electoral, con un titular que no podía ser más elocuente en esta polémica:

"Cádiz y la provincia, a consecuencia de una equivocada y lamentabilísima abstención de un importante sector de izquierda, da el triunfo a los ocho candidatos de derechas" (18).

Estos, también lo debieron entender así, porque no repararon en medios para favorecer la inhibición ante las urnas de los anarcosindicalistas, aunque fuera por procedimientos escasos de moralidad y de ética política.

Cuenta Stanley Payne, en su libro sobre la Falange, que Ramón de Carranza, el "gran cacique" de las derechas monárquicas gaditanas, no dudo en asegurar el abstencionismo anarquista, sobornando a algunos dirigentes sindicalistas, en un extraño pacto que el propio José Antonio Primo de Rivera quiso recrear en una novela político-humorística, que comenzó a escribir en inglés, con el título de Los anarco-carrancistas (19) Acción que también recoge Felipe Ximénez de Sandoval, uno de los biógrafos del líder falangista:

"Había bastado que alguien, que sabía lo que se hacía desde un punto de vista muñidor y electorero subvencionara a algún dirigente de la CNT, para que ésta acentuase su táctica inhibicionista" (20).

(15) BRENAN, Gerald: El laberinto español. Antecedentes sociales y politicos de la Guerra Civil. Barcelona, 1.977, pp. 323-325.

(16) ROBINSON, Richard: Los origenes de la España de Franco. Derecba, República y Revolución., 1.931-1.936. Barcelona, 1.977, pp. 323-325.

(17) MACARRO, José Manuel: La Utopia Revolucionaria. Sevilla en la Segunda república. Sevilla, 1.985 , pp. 366-367.

(18) El Noticiero Gaditano (20-XI-1.933).

(19) PAYNE, Stanley: Falange. Historia del fascismo español. París, 1.965, p. 38.

(20) XIMENEZ DE SANDOVAL, Felipe: José Antonio (Biografia apasionada). Madrid, 1.974, pp. 125-126. 
Pero descendamos a los municipios y veámos si existió o no esta relación entre el fenómeno abstencionista y la victoria derechista. Como puede verse en el CUADRO III, el triunfo de las candidaturas conservadora y radical se produce prácticamente en casi todos los municipios que superan el 60 por ciento de abstención, incluido los grandes núcleos urbanos de la provincia, como Cádiz, Jerez, San Fernando, Algeciras, Arcos o Sanlúcar, mientras que -al contrario-, en las cinco poblaciones donde se alcanzan los índices de participación más elevados, en todos gana la candidatura de la coalición republicanosocialista. Sin embargo, quizá sea alguien tan poco sospechoso como el ya citado José Antonio Primo de Rivera -diputado electo por las derechas de Cádiz en estas elecciones-, el que mejor vio esta relación, en un artículo que la censura no le dejó publicar, titulado "La victoria sin alas":

"En muchos sitios los obreros no han votado. Se han permitido el lujo escalofriante de regalar a la burguesía -a la derecha principalmente- la máquina de legislar. Una orden dada a tiempo por los sindicatos, una movilización general de las masas proletarias, hubiera producido la derrota de quién sabe cuántos candidatos de las derechas. Los obreros lo sabían y sin embargo, se han abstenido de votar" (21).

\section{PARTICIPACION ANARCOSINDICALISTA Y DERROTA DE LAS DERECHAS}

La victoria del Partido Radical y la CEDA en 1933 inauguró lo que más tarde la historiografia ha llamado el bienio "negro" o conservador, que terminó inesperadamente a finales de 1935, cuando los escándalos financieros que protagonizaron destacados políticos radicales, provocaron el descrédito de esta fuerza política y una crisis de Gobierno que se saldó con la disolución de las Cortes y la convocatoria de nuevas elecciones para el 16 de febrero de 1936.

La bipolarización de la vida pública, acentuada tras el Octubre asturiano del 34 y sus cientos de prisioneros, se traduce en estos comicios en la presentación en Cádiz -y en casi todas las circunscripciones- de dos candidaturas abiertamente enfrentadas: la de las derechas, con el Partido Radical dentro, bajo la denominación de Frente Antirrevolucio-

(21) DAVILA, Sancho-PEMARTIN, Julián: op. cit. p. 54. 
nario y la de la coalición de izquierdas, con el nombre de Frente Popular de Izquierda, más algún que otro candidato aislado de escasa relevancia electoral.

¿Cuál sería ahora la actitud del anarcosindicalismo gaditano ?. Pues bien, mientras que en Zaragoza, por ejemplo, la CNT había dado libertad de votos a sus afiliados, que en principio parecían inclinados a votar al Frente Popular para conseguir una amnistía que prometía sacar de las cárceles a los presos políticos (22), en Cádiz, esta calculada ambigüedad cenetista desaparece desde un primer momento, para dejar abiertamente paso a una posición beligerante en favor de las izquierdas. Habían ocurrido muchas cosas para que la CNT mantuviera las tesis abstencionistas de 1933, como reconocía un destacado miembro de su Comité Nacional en un mitin:

"Caer en la torpeza de hacer campaña abstencionista equivale a fomentar un triunfo de las derechas. Y todos sabemos por amarga experiencia de dos años de persecución lo que las derechas quieren hacer. Si el triunfo de las derechas se diera, yo os aseguro que aquella feroz represión a que sometieron a Asturias, se extendería por toda España" (23).

Pero más claro todavía era el destacado líder cenetista de Paterna, Miguel Pérez Cordón, en un artículo publicado en el periódico libertario La Tierra, a principios de 1935:

"(...) Dejemos al pueblo que hable. Escuchémoslo. Vamos de mal en peor. Combatimos los defectos de la República y sus hombres. Le hicimos la propaganda gratis a los monárquicos. Ahora no podemos hablar.

(...) Se pagan jornales de tres pesetas por lo que antes valía cinco o más. Trabajamos más.

\section{(...) Otro campesino nos manifiesta:}

-Es imposible ir por aceitunas y por bellotas. ¡Cuántas palizas han dado este año!" (...).

$\mathrm{Y}$ a unos y otros les pregunto:

-¿Qué haréis cuando lleguen otras elecciones?

-Votaremos.

-¿Votaréis?.

(22) BUENO, Jesús y otros: Elecciones en Zaragoza capital durante la II República. Zaragoza, 1.980 , p. 184.

(23) LORENZO, César M.: Los anarquistas españoles y el poder. París, 1.972, p. 72. 
-Sí, votaremos. Y que nadie venga diciendo lo contrario

-¿Por qué no votasteis en las elecciones de noviembre?.

-Hombre, porque sentimos mucho lo de Casas Viejas y otras cosas, y, además, creíamos que se implantaría el comunismo libertario(...).

$-i Y$ por quién votarás?- Les pregunto uno a uno.

-Por las izquierdas.

Me dan a entender que con las izquierdas habrá Casas Viejas, pero no jornales de hambre; sindicatos clausurados, pero no las organizaciones al margen de la ley; disparo sin aviso previo, pero no pena de muerte.

Aclaran:

-Entre un mal y otro hay que escoger. Lo menos malo son las izquierdas.

(...) Bueno; si la CNT os aconseja la abstención...

No me dejan terminar:

-¡Votaremos!" (24).

Y por si todavía había dudas, el 2 de febrero tenía lugar un mitin en Cádiz, "contra la pena de muerte, el fascismo y las leyes represivas", en el que intervenían juntos Rafael Calbo, presidente provincial del PSOE y candidato socialista en las listas del Frente Popular, que lo hacía en representación de la UGT y Vicente Ballester, líder del anarcosindicalismo gaditano, en una más que clara expresión del apoyo cenetista a la coalición de izquierdas.

Con este giro radical en la táctica del anarcosindicalismo, no podía extrañar que el día de las elecciones, el 16 de febrero, la prensa se hiciera eco de la "cantidad extraordinaria de electores" que formaban colas dispuestos a votar y que conocidos los resultados, la victoria del Frente Popular fuera indiscutible, obteniendo los ocho escaños reservados a la mayoría.

De un censo de 273.926 electores, habían acudido a las urnas 163.004 votantes, es decir, el 59,5 por ciento. El hecho de que este porcentaje sea nuevamente una de los más bajos de España, ha llevado a Tuñón de Lara a colocar a Cádiz como ejemplo -junto a Sevilla y Málaga-, de que "el voto de los anarquistas es importante, pero no decide los resultados", afirmando a continuación, que en estos distritos, "la mayoría de los cenetistas no votó" (25).

(24) Recogido en G. BREY-J. MAURICE: Historia y leyenda de Casas Viejas. Bilbao, 1.976, pp. 175-178.

(25) TUÑON DE LARA, M.: La crisis de ... P. 215. 
No compartimos esta opinión, por lo menos en el caso de Cádiz, porque como ya señaló Javier Tusell hace algunos años, quizá sea ésta la circunscripción electoral en donde más claramente se puede apreciar cómo la participación del anarcosindicalismo inclinó decisivamente los resultados en favor de las izquierdas. Estas ven casi triplicarse sus votaciones, gracias a que el electorado cenetista mayoritariamente acudió a las urnas.

Tusell hace un cáculo elemental, comparando los resultados de las elecciones de 1933, con las de ahora del 16 de febrero que dan la victoria al Frente Popular, que no puede ser más contundente:

\section{$1933 \quad 1936$}

$\begin{array}{lll}\text { Votantes } & 98.654 & 163.004 \\ \text { Izquierdas } & 30.967 & 98.437 \\ \text { Derechas } & 65.425 & 64.325\end{array}$

FUENTE: "Las elecciones del Frente Popular" Historia 16 , febrero, 1977, no 10 , p. 44.

Como puede observarse, mientras que las derechas solamente pierden 1.100 votos respecto a 1933 , es la candidatura frentepopulista la que recoge la casi totalidad de los 64.350 nuevos sufragios de los electores que acuden ahora a las urnas y que con casi toda seguridad se habían abstenido en 1933, produciéndose una subida de más de veinte puntos en el índice de participación, que pasa del 37,2 al 59,5 por ciento.

Pero por si estos resultados totales del conjunto de la provincia no son más que suficientes para demostrar nuestra hipótesis inicial sobre la decisiva influencia que tuvo el comportamiento de la CNT en las elecciones, vamos ahora a descender a los 15 municipios más abstencionistas de 1933 y veámos qué tipo de resultados ofrecen.

Como puede comprobarse en el CUADRO III, las dos conclusiones que podemos extraer no hacen sino completar todo lo que venimos diciendo. En primer lugar, si en 1933 en 8 de estas 15 poblaciones había ganado la derecha y en 3 los radicales, ahora en 1936, en 14 de las 15, la victoria corresponde al Frente Popular y, además, con unos resultados aplastantes, como ocurre en La Línea, Paterna, Ubrique, Arcos, Medina o Algeciras. 


\section{CUADRO III \\ RESULTADOS COMPARADOS DE 1933 Y 1936}

RESULTADOS DE 1933 RESULTADOS DE 1936

\begin{tabular}{lrrrrc} 
MUNICIPIO & $\mathrm{D}$ & $\mathrm{R}$ & $\mathrm{I}$ & $\mathrm{FA}$ & $\mathrm{FP}$ \\
Paterna de Rivera & 107 & 108 & 20 & 174 & 610 \\
El Gastor & 76 & 89 & 106 & 215 & 545 \\
Castellar & 53 & 56 & 31 & 91 & 291 \\
Los Barrios & 193 & 316 & 268 & 386 & 1.756 \\
Alcalá de los Gazules & 838 & 205 & 178 & 819 & 1.032 \\
Algeciras & 725 & 853 & 610 & 1.054 & 6.167 \\
San Roque & 179 & 322 & 471 & 462 & 3.148 \\
Sanlúcar & 2.344 & 600 & 243 & 4.412 & 1.891 \\
Bornos & 377 & 207 & 222 & 741 & 1.390 \\
Medina & 849 & 137 & 496 & 1.278 & 2.048 \\
La Línea & 489 & 1.091 & 2.378 & 926 & 8.394 \\
Arcos & 1.450 & 421 & 274 & 1.798 & 3.626 \\
Grazalema & 535 & 63 & 19 & 473 & 959 \\
Jimena & 819 & 376 & 350 & 1.038 & 1.547 \\
Ubrique & 629 & 351 & 184 & 647 & 1.889 \\
D=derecha & I=izquierda R=radical \\
FP=Frente Popular de Izquierda. & FA=Frente Antirrevolucionario \\
\multicolumn{7}{c}{ La segunda conclusión es más perceptible en el CUADRO IV: los } \\
29.282 votantes que acuden de más a las urnas sobre los de 1933, lo \\
hacen para apoyar prácticamente sin fisuras, a la lista del Frente Popular, \\
que recibe todos estos votos y un pequeño porcentaje más, que proba- \\
blemente procedería del electorado radical de 1933, que ahora se había \\
repartido entre las dos únicas candidaturas completas que se presentaban.
\end{tabular}

\section{CUADRO IV}

LOS NUEVOS VOTANTES DE 1936 EN LOS 15

MUNICIPIOS MAS ABSTENCIONISTAS DE 1933

$\begin{array}{lccc} & \mathbf{1 9 3 3} & \mathbf{1 9 3 6} & \text { DIFERENCIA } \\ \text { Votantes } & 20.525 & 49.807 & 29.282 \\ \text { Derecha } & 9.653 & 14.514 & 4.861 \\ \text { Izquierda } & 5.850 & 35.293 & 29.443 \\ \text { Radicales } & 5.195 & - & \end{array}$


Es evidente, como conclusión, que en Cádiz se había dado la curiosa paradoja que una fuerza en teoría antipolítica por anarquista, como la CNT, había decidido con sus actitudes -abstención y participación respectivamente- los resultados de las elecciones de 1933 y 1936 , gracias a su más que consolidada hegemonía en el seno del movimiento obrero gaditano. Con su "activa inhibición" le dio la victoria a las derechas monárquicas y con su participación a las izquierdas del Frente Popular. 